\title{
The nature and development of experts' strategy flexibility for solving equations
}

\section{Citation}

Star, Jon R., and Kristie J. Newton. 2009. The nature and development of experts' strategy flexibility for solving equations. ZDM - The International Journal on Mathematics Education, 41, 557-567.

\section{Published Version}

doi:10.1007/s11858-009-0185-5

\section{Permanent link}

http://nrs.harvard.edu/urn-3:HUL.InstRepos:4889493

\section{Terms of Use}

This article was downloaded from Harvard University's DASH repository, and is made available under the terms and conditions applicable to Other Posted Material, as set forth at http:// nrs.harvard.edu/urn-3:HUL.InstRepos:dash.current.terms-of-use\#LAA

\section{Share Your Story}

The Harvard community has made this article openly available.

Please share how this access benefits you. Submit a story.

\section{Accessibility}


Running head: EXPERTS' FLEXIBILITY

\title{
The Nature and Development of Experts' Strategy Flexibility for Solving Equations
}

\author{
Jon R. Star \\ Harvard University
}

Kristie J. Newton

Temple University

Paper submitted to the special issue of ZDM - International Journal of Mathematics Education

original submission: September, 2008

revised submission: February 2009

Correspondence should be addressed to: Jon R. Star, Harvard Graduate School of Education, 442 Gutman Library, 6 Appian Way, Cambridge, MA, 02138, 01-617-496-2511 (voice), 01-617-4963095 (fax), jon_star@harvard.edu.

Acknowledgements. Thanks to Martina Olzog, Jennifer Rabb, and Nira Gautam for their help in coding and analyzing the data reported here. This project was supported by a grant from Temple University to the second author. 
Running head: EXPERTS' FLEXIBILITY

\section{The Nature and Development of Experts' Strategy Flexibility for Solving Equations}




\section{Abstract}

Largely absent from the emerging literature on flexibility is a consideration of experts' flexibility. Do experts exhibit strategy flexibility, as one might assume? If so, how do experts perceive that this capacity developed in themselves? Do experts feel that flexibility is an important instructional outcome in school mathematics? In this paper, we describe results from several interviews with experts to explore strategy flexibility for solving equations. We conducted interviews with eight content experts, where we asked a number of questions about flexibility and also engaged the experts in problem solving. Our analysis indicates that the experts that were interviewed did exhibit strategy flexibility in the domain of linear equation solving, but they did not consistently select the most efficient method for solving a given equation. However, regardless of whether these experts used the best method on a given problem, they nevertheless showed an awareness of and an appreciation of efficient and elegant problem solutions. The experts that we spoke to were capable of making subtle judgments about the most appropriate strategy for a given problem, based on factors including mental and rapid testing of strategies, the problem solver's goals (e.g., efficiency, error-free execution, elegance) and familiarity with a given problem type. Implications for future research on flexibility and on mathematics instruction are discussed. 


\section{The Nature and Development of Experts' Strategy Flexibility for Solving Equations}

Success in algebra has been and continues to be a concern among educators and policy makers because of its important role as a gateway to college (National Mathematics Advisory Panel, 2008). Increasingly, proficiency with algebra and mathematics in general is considered to involve more than just skill; it involves an integration of skill and understanding that allows for flexible, adaptive, and appropriate use of algorithms, all of which contribute to efficiency, problem solving, and transfer of ideas to new situations (Baroody \& Dowker, 2003; National Research Council, 2001)). Yet, research on flexibility and how it develops is only emerging, particularly in the postelementary years. Existing research suggests that flexibility can be enhanced through appropriate instruction (Blöte, Van der Burg, \& Klein, 2001; Rittle-Johnson \& Star, 2007; Star \& Seifert, 2006), but much more research is needed to fully understand the development of flexibility.

Largely absent from the emerging literature on flexibility is a consideration of experts' flexibility. If researchers are to fully understand the trajectory of flexible problem solving in school mathematics, then it seems important to examine experts' approaches to advanced school topics such as algebra. Do experts exhibit strategy flexibility with algebra, as one might assume? If so, how do experts perceive that this capacity developed in themselves? Do experts feel that flexibility is an important instructional outcome in school mathematics? In this paper, we describe results from several interviews with experts to explore strategy flexibility for solving equations. 


\section{Strategy Flexibility}

Flexibility, particularly in terms of flexible use of strategies, is not a construct that has been consistently defined by researchers. Verschaffel, Luwel, Torbeyns, and Van Dooren (2007) suggest that researchers have conceptualized flexibility in a variety of ways. Some distinguish flexibility from adaptability, while others equate the two terms (Verschaffel, Luwel, Torbeyns, \& van Dooren, 2007). In addition, some researchers use the term to refer to a person's ease in switching between solution methods, whereas others also include a person's tendency to select the most appropriate method in a given situation. A further complication is that there are differing conceptions of what it means for a method to be most "appropriate;" some scholars focus primarily on efficiency and ease of execution as criteria for appropriateness while others includes additional task, environmental, or individual characteristics in making this determination (Verschaffel et al., 2007). Even the term "efficiency" is not particularly well-defined; the relationship between efficiency and other descriptors that may be applicable for evaluating strategies, including optimal, elegant, and best, is somewhat unclear, typically not based on behavioral data, and thus possibly quite subjective.

We choose to navigate through this somewhat confusing terrain by drawing heavily upon the work of Star, defining flexibility as knowledge of multiple solutions as well as the ability and tendency to selectively choose the most appropriate ones for a given problem and a particular problem-solving goal (Star, 2005; Star \& Rittle-Johnson, 2008; Star \& Seifert, 2006). We assert that flexibility exists on a continuum; as students gain flexibility they might first show increased knowledge of multiple strategies, then particular preferences, and finally appropriate use of preferred strategies (Blöte et al., 
2001). We use the term "appropriate" to refer to the strategy that is the most efficient, meaning that the strategy requires the fewest computational steps to execute. In addition, we treat the terms "best," "most efficient," and "optimal" as if they were synonymous to "appropriate."

Several recent studies have examined the development of flexibility among school-aged learners, and this work has identified promising instructional interventions that appear to improve students' flexibility. For example, Blöte and colleagues (2001) examined the impact of two instructional programs on 206 second graders' flexibility. One program emphasized conceptual understanding along with skill while the other program was more traditional, emphasizing standard procedures. Blöte and colleagues found that students in the conceptually-based program showed more flexibility in their preference and use of procedures, but use lagged behind preference in both groups. They also suggested that students who used only one procedure for a prolonged length of time had more difficulty adopting new procedures.

Similarly, Star and Seifert (2006) investigated the impact of an instructional intervention -- having students solve the same problem in two different ways -- on students' flexibility for solving algebraic equations. Specifically, students in an experimental group $(n=18)$ were asked to solve previously completed problems in new and different ways, while students in a control group $(n=18)$ solved a series of distinct problems. The researchers found that, while the groups performed similarly with regard to accuracy, students in the treatment group were more likely to use multiple strategies and to invent new strategies for solving equations. 
Rittle-Johnson and Star (2007) extended this work by examining a closely related instructional approach, that of explicitly comparing solution methods. Students worked in pairs to study and answer questions about pre-worked examples. The treatment group (n $=18$ pairs) studied the same problem worked two different ways and answered questions that encouraged comparison. The control group ( $\mathrm{n}=17$ pairs) studied the same two methods, but used isomorphic problems to do so, and the methods were studied sequentially. At posttest, the two groups had gained similarly with regard to conceptual knowledge, but the treatment groups had greater gains in both procedural knowledge and flexibility.

Studies such as these are promising and inform the field about instructional interventions that appear to promote students' flexibility. However, a more complete understanding of flexibility and its development requires an exploration of the flexibility of proficient solvers. If flexibility is an important component of students' proficiency in mathematics (Kilpatrick et al., 2001), it seems critical to have more in-depth knowledge of what this capacity looks like in experts. Do experts exhibit flexibility? How and when do experts become flexible? As discussed below, the literature on experts' flexibility is quite limited and yields inconsistent results. (Note: Drawing upon the cognitive research on expertise, we use the term "experts" here to refer to individuals with significant and deep knowledge in a domain, as a result of substantial practice extending over a minimum of 10 years (Ericsson \& Charness, 1994; Ericsson, Krampe, \& Tesch-Romer, 1993).) 


\section{Experts and Flexibility}

Although many studies have examined differences between experts and novices with regard to complex academic tasks (particularly in physics; Chi, Feltovich, \& Glaser, 1981; Larkin, McDermott, Simon, \& Simon, 1980), few have focused specifically on strategy flexibility in mathematics. The limited research suggests that experts do tend to have multiple, efficient strategies for solving problems, but findings are inconsistent about the extent to which experts employ these strategies.

For example, in order to understand the strategies that experts use to estimate products and quotients, Dowker (1992) gave 44 mathematicians randomly ordered estimation tasks and recorded their responses. 18 of them were retested several months later and the strategies were compared. The mathematicians not only exhibited a high level of accuracy, but they used a variety of strategies, and the strategies tended to illustrate their knowledge of number properties and relationships. In other words, experts' strategies demonstrated both flexibility and conceptual knowledge.

Similarly, Cortés (2003) noticed a link between experts' approach to solving problems and their understanding of mathematical structures. He interviewed five high school mathematics teachers, ten engineers and scientists, and ten high school students to understand their knowledge of and approach to solving algebraic equations, inequalities, and systems of equations. Cortés found that the experts were not only efficient, but they rapidly analyzed the task and decided on an approach based on the characteristics of the problem. Students, on the other hand, were more likely to attach a particular algorithm to a particular kind of problem, and they were less likely to consider any justification of their steps. 
In contrast to these findings, Carry and colleagues (Carry, Lewis, \& Bernard, 1979) reported that the primary difference between more and less able solvers was not that experts knew and used a greater number of strategies but rather that more able solvers tended to make fewer errors. These researchers observed how two groups of college students solved algebraic equations. One group $(n=19)$ consisted of volunteers from an introductory psychology course who were expected to be poor equation solvers, and one group $(n=15)$ consisted of engineering and mathematics students who were expected to be good equation solvers. All students were asked to complete an algebra test while commenting on the decisions they made. Carry and colleagues found that experts tended to make fewer errors, but the two groups showed only small differences in the number of and types of strategies that were employed.

In a follow-up study, Lewis (1981) gave the same test to more proficient solvers and compared the results to the ones reported above. Specifically, five working mathematicians took the same test that was administered in Carry et al. (1980). Results suggest that although these experts tended to use more efficient strategies than either group in the prior study, experts' problem-solving accuracy was not strikingly different than the more able students in Carry et al.

These and other studies (e.g., see Krutetskii, 1976; Wertheimer, 1959, for early examples) provide a useful starting point for understanding experts' flexibility. However, there are several key weaknesses to this literature that suggest the need for additional studies. First, existing research does not explore the development of flexibility. An important step in creating and evaluating instructional interventions to promote flexibility is to identify the conditions by which experts' flexibility emerged. Recent research 
suggests that instruction can influence flexibility (Blöte et al., 2001; Rittle-Johnson \& Star, 2007; Star \& Seifert, 2006). Do experts attribute their flexibility to prior instruction? If not, how did they become flexible problem solvers? Do they believe instruction has a role in developing flexibility? Clearly, more research is needed about how experts evolve into flexible solvers.

Second, existing research does not sufficiently explore the nature of experts' flexibility. In particular, research does not clearly distinguish between experts' use of algorithms, knowledge of alternate methods, and preference for particular strategies. Work from Star and Rittle-Johnson (in press) suggests that students may know multiple strategies but choose to use a single strategy for a given problem type. Similarly, Blöte and colleagues (2001) demonstrated that young learners begin to show preferences for alternate algorithms before they regularly begin to use them. In addition, work by Van Dooren and colleagues indicates that prospective elementary school teachers prefer algebraic strategies, even with arithmetic methods are more straightforward (Van Dooren, Verschaffel, \& Onghena, 2002, 2003). Are these patterns true of experts? Do experts consistently use the strategies they prefer or do they sometimes use standard algorithms despite knowledge of and preference for alternate ones? Research from (Carry et al., 1979) suggests that proficient solvers do not regularly use more efficient strategies, but the participants in their study were not asked if they knew of alternate ways to solve the problems or whether they had a preference in how to solve the problems; they were simply asked to comment on the strategies they chose to use. Consequently, studies that focus on when and why experts deviate from standard algorithms, as well as why and under what circumstances they prefer alternate approaches are lacking. 
Finally, the aforementioned expert studies have provided limited opportunities for experts to demonstrate flexibility. Although some problems that allow for efficient or elegant solutions were included, the primary purpose of the studies was to understand experts' strategies and accuracy in general, not to explore flexibility per se. As a result, they were given few problems that were explicitly designed to test strategy flexibility. More studies need to include assessments that are explicitly designed to explore flexibility in experts.

\section{Current Study}

The current study attempts to address the above weaknesses in the literature on experts' flexibility. Specifically, we designed tasks that provided opportunities for experts to demonstrate flexibility; we probed experts about the approaches they noticed and preferred; and we asked experts to reflect on the emergence of this capacity in their own learning. Similar to Cortés (2003), we explored flexibility among experts from several different fields, including mathematics, mathematics education, engineering, and secondary mathematics instruction. Our analysis of experts' interviews and problem solving focuses on the following issues.

First, we are interested in the nature of experts' flexibility for solving algebra problems. As noted above, prior research has shown that, while experts are less likely to exhibit errors in problem solving than novices, the degree to which experts show knowledge of multiple strategies and the ability to adaptively select the most appropriate strategy has varied across studies. Our investigation of experts' flexibility will focus on several aspects of strategy choice, including use of multiple strategies, knowledge of multiple strategies, and preferences for certain strategies. A second focus of the current 
work is development of experts' flexibility developed. We believe that this developmental perspective on flexibility among experts has not been well-explored in prior research. Related issues include whether experts believe that flexibility is an important outcome and how experts believe flexibility can or should be fostered in school mathematics.

As such, several research questions guided our study. To what extent do experts use multiple approaches and adaptively select the most appropriate strategies for a given problem? How do experts select problem solving strategies? Do experts hold preferences for particular strategies for certain problems, and, if so, what are these preferences based on? How do experts become flexible, and what are their views about the role of instruction in developing flexibility?

\section{Method}

\section{Participants}

Participants for the current study included eight experts in school algebra. Specifically, a convenience sample of two mathematicians, two mathematics educators, two secondary mathematics teachers, and two engineers were included in the study. One of the mathematicians, (Mark; pseudonyms are used for all experts), held a doctorate in mathematics and had worked for years in a university mathematics department. The other mathematician (Matthew) was finishing his doctorate in mathematics but had also taught high school mathematics and worked with teachers for many years. Both mathematics educators held masters degrees in mathematics; one also held a doctorate in educational studies with a concentration in mathematics education (Evelyn), and the other was finishing her doctorate in mathematics education at the time of data collection (Ellen). One of the mathematics teachers was a veteran secondary teacher with a bachelors degree 
in physics and a masters in education with a mathematics emphasis (Tara). The other teacher held degrees in psychology and sociology (Timothy) but had been a professional tutor for many years and often taught high school mathematics in the summer. One of the engineers held bachelors degrees in mathematics and in aerospace engineering (Nathan). His job included writing flight simulations for rockets and satellites, which involved extensive use of algebra, calculus, and other areas of mathematics. The other was a mechanical engineer, with both a bachelors and a masters degree in the field. His job involved designing and testing electrical tools (Nicholas). These types of experts were chosen in order to provide a range of perspectives on flexibility and potentially different approaches to solving problems. Participants were from five different states, all within in the eastern United States. Five of the experts were male and three were female. Consent forms were secured for all participants.

\section{Measures}

Opportunities to assess flexibility were embedded in a researcher-designed algebra test, which included many items with only one apparent solution method. This approach was used to ensure participants did what naturally occurred to them; if all problems could be solved in multiple ways, then it may prompt participants to search for the best methods. The 55-item test was originally designed to be used as a final examination for a three-week summer course for high school students that reviewed more advanced topics from a first year of school algebra. Test items were symbolic mathematics problems taken or adapted from a standard algebra text on solving and graphing both linear and quadratic equations, as well as simplifying expressions with exponents and square roots. The exam was adapted from prior exams created by a veteran 
high school teacher, with edits made to ensure opportunities to demonstrate flexibility. For example, one problem in the form $a(x+b)=c$ was altered such that $c$ was divisible by $a$. Namely, $7(n+13)=42$ was included in the assessment. Whereas students are often taught to distribute first for equations that include parentheses, dividing both sides of the equation by 7 is an alternative first step to solving this equation. The complete assessment is included in the Appendix.

Semi-structured interviews were conducted by the second author to probe experts' thinking about their strategies for solving algebraic problems. Experts were asked to explain how they solved a subset of problems on the assessment, why they chose the strategies they used, whether they knew of other ways to solve the problems, and which strategies they preferred. Experts were also asked to elaborate on issues of interest as they emerged; in particular, we probed what experts meant when they used terms to describe strategies such as easy, better, best, elegant, efficient, neat, etc. Problems were selected based on their possible relevance for exploring issues of flexibility. For example, experts were asked to explain their strategy on problem $\# 24$, which asked them to simplify $\sqrt{\frac{75}{3}}$. Because $\frac{75}{3}$ simplifies to a perfect square, simplifying first might be considered an efficient alternative to taking the square root of the numerator and the denominator separately (a standard approach for roots involving fractions). After explaining their thinking on these problems, experts were given a brief introduction to the authors' conceptualization of flexibility and then asked about own experiences that led to becoming flexible and whether they thought flexibility should be or could be taught in schools. 


\section{Procedure}

The algebra test was administered in a one-on-one setting to the experts at a time and place convenient to them. At the beginning of the test administration, experts were told that the focus would be on the methods they used to solve the problems. As such, showing work was encouraged. They were informed that an interview would follow the test in which they would be asked about selected problems, but they were not told in advance which particular problems would be selected. The test was not timed, but most of the experts completed it within 20 minutes. A few took longer, either because they could not immediately remember a particular formula and hence had to derive it, or because they were being extra careful to check their work. Participants were compensated for their participation with a gift card to a local bookstore.

The interviews were conducted immediately following completion of the test. Interview times varied, ranging from about 10 to 30 minutes. Variations were primarily a result of some experts giving brief explanations, such as stating they used the distributive property, and some experts giving detailed explanations, such as specifying the particular number they distributed and what products resulted. Interviews were audiotaped and subsequently transcribed.

\section{Coding}

The transcribed interviews were listened to multiple times for analysis purposes. Three graduate students assisted in the analysis and met regularly with the authors to discuss findings and resolve issues regarding interpretation. The second author, who had conducted the interviews, served as a check on this process. Analysis proceeded in three parts. First, solution methods were examined for evidence of knowledge of and flexible 
use of multiple strategies. Similarities and differences across experts were noted. Second, experts' rationales for strategy choices were examined for patterns, and common themes were noted. Third, explanations of how flexibility emerged were categorized.

\section{Results}

We begin by reporting results on the nature of experts' flexibility, followed by an analysis of experts' views on how their flexibility developed.

\section{The Nature of Experts' Flexibility}

It is important to first note that the eight experts interviewed in this study were quite successful on the mathematics tasks that they were asked to complete. Consistent with prior work on experts in mathematics and other domains, our experts rarely made errors. When errors were made, experts quickly noticed and corrected them.

Our interest, however, was more on the strategies that our experts used and their flexibility. We found that the experts interviewed were quite flexible. They exhibited knowledge of and use of multiple strategies for solving a range of problems, and they generally used and/or expressed a preference for the most efficient strategies for a given problem.

Choice of strategies. We first consider the criteria that our experts used to select their chosen strategy (which was often the most efficient strategy) for a given problem. Overwhelmingly, our experts indicated a preference for strategies that they deemed to be easiest. In general, the easiest strategy was the one that was identified by experts as "faster, quicker, less steps" (Tara). However, fewer steps was not the only consideration for choosing an easy strategy; reducing effort also appeared to be important. As one 
expert suggested, “It's not about extra steps. I don't mind putting in extra steps if extra steps makes it easier." (Nathan). Referring to problem \#27, 7(n+13) = 42, one expert noted, "I wanted to minimize the effort. So another way to do it is distribute, but I didn't want to do that" (Matthew). Another stated, "You can distribute, make your life a little harder...because you have to play with bigger numbers" (Mark).

Our experts also referred to the "neatness" of a strategy in explaining their strategy choices. For example, one expert noted that to solve problem \#27, he felt it was best to use division by seven as the first step because "That was evenly divisible. If it wasn't divisible it wouldn't get a nice, clean answer" (Nicholas). Similarly, another expert also noted that, "Distributing, I would have had to deal with fractions and finding common denominators and things wouldn't have been as nice" (Tara). As these examples suggest, neatness for these experts appeared to indicate that the next step of the problem involved integers.

Avoiding fractions was of particular interest to many of the experts. One expert explained that she did not like to work with fractions because they are "slower to operate with" (Ellen). In general, reducing the arithmetic complexity of the problem was important for both speed and accuracy, according to our experts. On expert explained, "I'm less likely to make a calculation error" (Ellen). Another expert concurred, stating that "I am not real quick at arithmetic, so I like to keep the arithmetic as simple as possible" (Evelyn).

In addition to selecting a strategy based on its perceived ease of execution, our experts also considered the specific characteristics (e.g., structure and coefficients) of problems in selecting a strategy. This criteria for selecting strategies was particularly 
noticeable when the experts solved systems of linear equations. For example, when solving problem \#36 (see Appendix), the system which included the lines $3 y+4 x=0$ and $y=x-7$, one expert explained "I used substitution because it was set-up that way" (Timothy). Presumably Timothy is referring to the fact that the second line is already written in the form $y=$ ?, which makes it particularly amenable to substitution in the other equation. Similarly, when another expert solved problem \#35, which is the system containing the lines $2 x+y=1$ and $x+y=3$, he explained that he used the addition/subtraction method because "you have to get rid of something and in this case you just observed that $y$ is the same, so subtraction will get rid of it" (Mark). Mark here refers to the fact that both equations were written in standard form, and the $y$ coefficients were equal, making subtracting a seemingly straightforward method to implement.

Our experts also relied on their own familiarity with problem types and strategies when determining the best approach for solving a given problem. For example, when solving problem \#39, $x^{2}-10 x+25$, one expert immediately recognized this trinomial as a perfect square, noting, "I just know the factorization is $x$ minus five, $x$ minus five in my head so I just rewrote $x$ minus five squared" (Tara). Referring to this same problem, another expert said, "It's a complete square. I recognize complete squares.” (Mark).

Failure to choose optimal strategy. Consistent with prior research (Carry et al., 1979), our experts did not always choose the most efficient strategy for a given problem. Typically, when experts failed to choose the optimal strategy and were subsequently asked about their strategy choice, they tended to note that they "weren't thinking." For example, one expert solved problem \#27, $7(n+13)=42$, by distributing the seven as his first step. When he later noticed that it would have been more efficient to divide both 
sides by seven first, he stated, "I just didn't think about it at the time. I just blew through it" (Nathan). In addition to "not thinking," other experts indicated that their initial choice of non-optimal strategies came because of well-practiced, automatized approaches that they initiated very quickly after seeing a problem. For example, another expert, who also used distribution as the first step to solve this same problem (\#27), explained his choice as, "For some reason I just went straight to the formula. I guess [I was] in that mindset" (Timothy). In general, experts explained that the reason for the less efficient method was usually a result of not looking carefully at the particular structure or coefficients of a problem.

However, our experts' rationales for their strategy choices clearly indicated their strong tendency to prefer efficient strategies even when these strategies were not used for solving a given problem. For problem $\# 33,1 / 3(x+5)+2 / 3(x+5)=7$, both Ellen and Nathan multiplied both sides of the equation by 3 in an attempt to avoid calculating with fractions. However, when asked if they knew of another way to solve the problem, both said a better first step would be to combine "like" terms, obtaining $x+5=7$, and both expressed a preference for this method. When asked why she preferred the second method, Ellen suggested, “There's something about recognizing that those two things equal one that I, I don't know, that I like....There is something pretty about it." After noticing this alternate approach, Nathan described his original strategy as "incredibly convoluted" in comparison, adding that, "In this case, my fixation on getting rid of the denominators kind of obscured the problem there." Mark referred to solutions that took advantage of particular characteristics as "clever." 


\section{Development of Experts' Flexibility}

Recall that toward the end of the interview, our experts were introduced to the construct of flexibility (as we conceptualize it here) and were asked to reflect on their flexibility and its development in their own learning in school and college mathematics classes. With the caveat that experts' self-reports of their own educational experiences are inherently biased and may not objectively reflect the actual intentions of their prior mathematics instructors, we report the results of experts' recollections below.

After learning about flexibility, not surprisingly our experts uniformly believed that they themselves were flexible. Furthermore, when considering how their own flexibility developed, our experts did not believe that flexibility was an overt instructional goal for their K-12 or university mathematics instructors. A typical response to being asked if they were taught flexibility was, "No, never!" (Evelyn). Instead, experts offered two explanations for how they developed strategy flexibility - both of which minimize the role of a teacher in imparting flexibility to his/her students.

First, several experts felt that their own flexibility had emerged as a natural consequence of exposure to seeing similar kinds of problems over and over again, combined with a desire to solve problems as quickly as possible. One expert stated, "My best guess is just a lot of repetition and when you to do small equations over and over and over you're going to want to find the quickest way of doing things to get done faster" (Tara). This desire to complete problems as quickly as possible could have been present because of disinterest (e.g., wanting to complete math homework as quickly as possible), but among those we interviewed, it seemed more common that experts found the search for the quickest and easiest strategies for given problems to be intellectually challenging 
and interesting. Experts did not report that their mathematics instructors had pushed them to search for optimal strategies, but rather that this was a desire that they themselves brought to problem solving. As one expert stated, "It's a challenge to solve them in different ways, but then you start to learn which methods are quickest or easiest for certain problems, and you notice certain patterns" (Nathan).

Another explanation that several experts favored was that their flexibility was the result of their teaching. As one expert explained, "I've taught this stuff. I guess when you teach kids you get to know ten different ways of doing it based on what kids like. And it got me ready to be able to respond to each of those different ways and not favor one way over another" (Matthew). Similarly, another expert said, "When you have taught this stuff enough, and I have, you get used to seeing it and answering questions" (Evelyn). And when describing how he developed flexibility, Timothy suggested, "I tutor students mainly who struggle, so I have just learned that if they can't see it one way, often trying another way helps them see something they didn't see before." In other words, through interactions with students -- both having to explain problems in multiple ways to struggling students and also by exposure to the idiosyncratic, original, or even erroneous strategies that students produce, experts developed more robust knowledge of multiple strategies for solving algebra problems.

Overall, the experts appeared to value flexibility and felt that it was an integral part of doing mathematics. As one expert described, "Problem solving is a general skill, and you have to be adaptable and flexible to the context" (Nathan). However, and consistent with experts' views on how their own flexibility developed, there was variation as to whether experts felt it was a good idea to teach students to be flexible. Some experts 
thought that teaching flexibility was a good way to teach students to notice the mathematical structure in problems and that it would help "the students understand the mathematics more deeply" (Evelyn). Yet others thought that teaching for flexibility would confuse students and that students should learn from trial and error. One expert stated, "You can learn some tricks from your teachers, but eventually it all comes down to doing it yourself" (Mark).

\section{Discussion}

The purpose of this study was to explore the strategy flexibility of experts. Despite the recent emergence of a literature on students' flexibility, relatively little research exists on experts' flexibility. In our interviews with eight experts, we explored the nature of experts' flexibility as well as experts' views on the development of this capacity. Our results indicated that our experts did exhibit strategy flexibility on the tasks that they were asked to complete: Experts showed knowledge of multiple strategies and the ability to select appropriate strategies for given problems. The experts that we interviewed expressed a strong preference for strategies that they deemed to be easiest, where the easiest strategies were those which resulted in the fewest steps, the least effort to execute, and/or the reduction of arithmetic complexity. Our experts also considered the specific characteristics of problems (including a problem's structure and its coefficients) when selecting a strategy. On the whole, experts preferred to use the strategy that they deemed most "compatible" with the characteristics of a given problem (Nistal, Van Dooren, Clarebout, Elen, \& Verschaffel, in press).

An interesting issue that seems worthy of future exploration is the complex and subtle relationships that likely exist between the various criteria that experts used to 
select strategies. For example, our experts expressed a preference for strategies that they determined to be easiest. To what extent are the easiest strategies those which also provide a reduction in arithmetic complexity? Or does such a connection depend on a solver's facility with (for example) fractions, such that some experts might find a strategy that eliminates fractions to be less efficient (e.g., more time consuming to execute)? Similarly, to what extent do concerns about accuracy coexist with a desire for efficiency? And again, might a solver's facility with arithmetic impact determinations of the relative efficiency of a strategy or its potential to be executed without error? We have begun to explore some of these questions in novice equation solvers (Newton \& Star, 2009), but it also seems important to learn more about how these strategy selection criteria interact among expert solvers.

Consistent with prior research, we found that our experts did not always select the optimal strategy for a given problem, despite their knowledge of and preference for the most efficient strategies. With respect to the development of their own flexibility, our experts did not believe that flexibility had been an overt instructional goal for their prior mathematics instructors. Rather, the experts interviewed here believed that their flexibility emerged from their own initiative and/or as a consequence of their teaching experiences.

Somewhat surprisingly, there was unanimity among the experts we spoke to in identifying the optimal strategy for a given problem. Prior research has indicated that students' views on what it means for a strategy to be optimal for a given problem are quite idiosyncratic and divergent (Star \& Madnani, 2004), yet the experts interviewed here were generally in consensus about the best strategy for a specific problem. Related, 
we expected some experts to argue that a well-practiced strategy that could be easily retrieved and implemented with minimal thought (such as distributing the 7 first on problem \#27, $7(n+13)=42)$ and without consideration of problem-specific features (such as the divisibility relationship between 42 and 7 in this problem), would be more efficient than taking a moment to examine a problem and then choose among known strategies to select the most one matched to the problem's specific features or structure. However, among the experts that we interviewed, this sentiment was not expressed; instead, experts expressed a clear preference for elegant strategies (e.g., strategies chosen to take advantage of a problem's unique characteristics).

Below, we discuss several implications of our results, for research on flexibility and for mathematics instruction more generally.

\section{Implications for Mathematics Instruction}

The experts interviewed here were in agreement that flexibility was not an explicit focus of their K-12 and university mathematics experience (as Evelyn noted, "No, never!"). One interpretation of these experts' views would be that flexibility should not be an instructional target in elementary and secondary schools - that flexibility is best developed implicitly and individually by the repeated problem solving experiences of learners. According to this interpretation, developing flexibility requires a significant amount of personal initiative, including the propensity to find the search for quicker and easier strategies to be an interesting and intellectually challenging endeavor. Thus flexibility would be considered as an advanced instructional goal - one that was only available to those with a great deal of talent in mathematics and an exceptional drive to develop this competency in themselves, largely without the aid of teachers. 
This point of view runs counter to the current emphasis in the US on the importance of flexibility for all students in K-12 mathematics instruction (e.g., National Mathematics Advisory Council, 2008; Kilpatrick et al., 2001). Flexibility is currently viewed as an outcome that is attainable and critical for students of all ability levels. A proponent of this current emphasis on flexibility for all might point out that much has changed in the years since these experts attended elementary and secondary school, including a greater variety of curricula, greater diversity in instructional methods, and more generally increased attention to providing quality mathematics instruction to all students. Improvements such as these suggest that, despite some experts' views to the contrary, it may be possible to consider flexibility as an instructional goal for all students, rather than as an outcome that is only available to future experts who pursue it themselves.

An alternative interpretation of our experts' views on the development of their own flexibility might be that these experts were providing a critique of the mathematics instruction that they received in their past. In other words, some experts may look back on their prior mathematics instruction and wish that it had been more focused on flexibility. This interpretation speaks to the difficulties of helping teachers to teach flexibility. It seems clear that, in order for teachers to foster flexibility in their students, the teachers themselves need to be flexible.

We recently confronted the challenges associated with instructing teachers about flexibility in a one-week professional development institute (Yakes \& Star, 2009). While our intent in the professional development was to help teachers foster flexibility in their students, it quickly became apparent that we first needed to address teachers' own 
flexibility. By discussing multiple strategies for solving a variety of algebra problems, teachers began to reflect on the ways that they did or did not include multiple strategies in their own instruction. As one teacher participant noted, "I know that when I was learning math, I often fell back on one way of solving a problem. I think this did not allow for a better understanding of the topic because I was so focused on one solution method. This one-way method put up a sort of roadblock in my understanding." Similarly, another teacher said, "I realize that intuitively I choose a method that is best/most efficient/easiest for me when I work on the board, but I have never taken the time to express why or even let the students suggest why."

These responses are consistent with recent research on prospective teachers' knowledge of fractions (Newton, 2008). After taking a course that helped deepen their conceptual knowledge of the mathematics they were to teach (in particular, their understanding of general algorithms), Newton found that there were no overall differences in prospective teachers' strategy flexibility. Exceptions included topics about which alternative methods were explicitly modeled or discussed. Newton's study suggests that flexibility needs to be a specific goal of teacher education and development professional development that focuses primarily on conceptual knowledge may not be particularly effective at promoting flexibility.

\section{Implications for Research on Flexibility}

This study underscores the importance in future research of using tasks that are specifically designed to elucidate flexibility. Prior work with students has shown that knowledge of innovative strategies for problem solving often precedes the ability to implement these strategies (Star \& Rittle-Johnson, 2008). Similarly, this study and others 
(e.g., Carry et al., 1980) suggests that even experts do not always use the most efficient strategy for solving a given problem. As a result, merely giving students a list of problems to solve may not be a good indicator of flexibility; students may choose to use the same strategy for all problems, even when it is not the most efficient choice, and despite their knowledge of alternative approaches.

In this study and in our prior work, we have used two kinds of tasks to more effectively assess students' flexibility. First, we have conducted interviews to accompany problem solving, asking participants to explain and justify their choices of strategies. And second, we have incorporated different kinds of problems into our assessments to better tap participants' flexibility. In some cases, we have merely changed problem coefficients, to accentuate the benefits of using an alternative strategy. For example, instead of using a problem such as $3(x+1)=22$, we might alter the problem to $3(x+1)=21$, to perhaps increase the likelihood that participants who were aware that they could divide both sides by 3 first would actually implement this strategy. In addition, we also devised other kinds of tasks to tap flexibility, including asking participants to solve the same problem in more than one way and to identify which strategy was optimal. It seems important for researchers to consider ways to include tasks such as these, as well as other innovative tasks and methodologies used by other researchers (e.g., the choice/no-choice paradigm; Siegler \& Lemaire, 1997), to adequately investigate strategy choices and the development of flexibility.

\section{Conclusion}

Strategy flexibility is an important instructional goal in mathematics instruction at all levels. An emerging research base on students' flexibility is beginning to provide 
helpful guidance on the development of flexibility and instructional tasks that reliably lead to greater flexibility. However, experts' flexibility has been relatively unexplored. This paper provides initial evidence about some experts' views on the nature, development of and importance of flexibility. Despite agreement among our experts that flexibility was not emphasized in their own learning of mathematics, experts in this study had a natural and pervasive tendency to value and use efficient, elegant strategies to solve algebra problems. This tendency seems to be deeply related to their knowledge of the subject, underscoring the need to include flexibility as a goal for instruction at the secondary level. 


\section{References}

Baroody, A. J., \& Dowker, A. (Eds.). (2003). The development of arithmetic concepts and skills: Constructing adaptive expertise. Mahwah, NJ: Lawrence Erlbaum.

Blöte, A. W., Van der Burg, E., \& Klein, A. S. (2001). Students' flexibility in solving two-digit addition and subtraction problems: Instruction effects. Journal of Educational Psychology, 93, 627-638.

Carry, L. R., Lewis, C., \& Bernard, J. E. (1979). Psychology of equation solving: An information processing study (Final technical report). Austin: The University of Texas. Document Number)

Chi, M. T. H., Feltovich, P. J., \& Glaser, R. (1981). Categorization and representation of physics problems by experts and novices. Cognitive Science, 5, 121-152.

Dowker, A. (1992). Computational estimation strategies of professional mathematicians. Journal for Research in Mathematics Education, 23(1), 45-55.

Ericsson, K. A., \& Charness, N. (1994). Expert performance: Its structure and acquisition. American Psychologist, 49(8), 725-747.

Ericsson, K. A., Krampe, R., \& Tesch-Romer, C. (1993). The role of deliberate practice in the acquisition of expert performance. Psychological Review, 100, 363-406.

Krutetskii, V. A. (1976). The psychology of mathematical abilities in school children (J. Teller, Trans.). Chicago: University of Chicago Press.

Larkin, J., McDermott, J., Simon, D. P., \& Simon, H. A. (1980). Expert and novice performance in solving physics problems. Science, 208(4450), 1335-1342.

Lewis, C. (1981). Skill in algebra. In J. R. Anderson (Ed.), Cognitive skills and their acquisition (pp. 85110). Hillsdale, NJ: Lawrence Erlbaum Associates.

National Research Council. (2001). Adding it up: Helping children learn mathematics. Washington, DC: National Academy Press.

Newton, K. J. (2008). An extensive analysis of elementary preservice teachers' knowledge of fractions. American Educational Research Journal, 45(4), 1080-1110.

Newton, K.J., \& Star, J.R. (2009). Exploring the development of flexibility in struggling algebra students. Under review.

Nistal, A. A., Van Dooren, W., Clarebout, G., Elen, J., \& Verschaffel, L. (in press). Conceptualising, investigating, and stimulating representational flexibility in mathematical problem solving and learning: A critical review. ZDM.

Rittle-Johnson, B., \& Star, J. R. (2007). Does comparing solution methods facilitate conceptual and procedural knowledge? An experimental study on learning to solve equations. Journal of Educational Psychology, 99(3), 561-574.

Siegler, R. S., \& Lemaire, P. (1997). Older and younger adults' strategy choices in multiplication: Testing predictions of ASCM using the choice/no-choice method. Journal of Experimental Psychology: General, 126(1), 71-92. 
Star, J. R. (2005). Reconceptualizing procedural knowledge. Journal for Research in Mathematics Education, 36(5), 404-411.

Star, J. R., \& Madnani, J. (2004). Which way is best? Students' conceptions of optimal strategies for solving equations. In D. McDougall \& J. Ross (Eds.), Proceedings of the twenty-sixth annual meeting of the North American chapter of the International Group for the Psychology of Mathematics Education (pp. 483-489). Toronto: University of Toronto.

Star, J. R., \& Rittle-Johnson, B. (2008). Flexibility in problem solving: The case of equation solving. Learning and Instruction, 18, 565-579.

Star, J. R., \& Seifert, C. (2006). The development of flexibility in equation solving. Contemporary Educational Psychology, 31(280-300).

Van Dooren, W., Verschaffel, L., \& Onghena, P. (2002). The impact of pre-service teachers' content knowledge on their appreciation of pupils' strategies for solving arithmetic and algebra word problems. Journal for Research in Mathematics Education, 33(5), 319-351.

Van Dooren, W., Verschaffel, L., \& Onghena, P. (2003). Pre-service teachers' preferred strategies for solving arithmetic and algebra word problems. Journal of Mathematics Teacher Education, 6(1), 27-52. Verschaffel, L., Luwel, K., Torbeyns, J., \& van Dooren, W. (2007). Developing adaptive expertise: A feasible and valuable goal for (elementary) mathematics education? Ciencias Psicologicas, 2007(1), 27-35. Wertheimer, M. (1959). Productive thinking (Enlarged ed.). New York: Harper \& Brothers.

Yakes, C., \& Star, J. R. (2009). Using comparison to develop teachers' flexibility in algebra. Under review. 


\section{Appendix}

\section{Algebra Assessment}

True or False

1. $50=1$

2. The number 110 is a perfect square

3. For all $x>0,(x+6)^{2}=x^{2}+36$

4. $25=52$

5. When $x \neq 0, \frac{0}{x}=0$

Fill in the Blank

6. The reciprocal of $\frac{2}{3}$ is

7. The slope of $y=6 x+\frac{3}{8}$ is

$8.5(y+3)=5 y+15$ demonstrates the _ property.

$\underline{\text { Simplify }}$

9. $\mathrm{m}^{4} \cdot \mathrm{m}^{3}$

10. $\left(3 a^{5}\right)^{2}$

11. $\frac{y^{8}}{y^{2}}$

12. $4 x^{2}+3 x-7 x+9 x^{2}+6 y$

13. $\sqrt{18}$

14. $\sqrt{3} \cdot \sqrt{30}$

15. $(x+4)(x-7)$

16. $4 x \cdot 4 x^{2} y$

17. $4 x\left(x+2 y^{3}\right)$ 
18. $\frac{10 x^{5} y^{3}}{4 x y^{7}}$

19. $\sqrt{8}+5+6 \sqrt{50}$

\section{Evaluate}

20. If $a=3$ and $b=-6$,

21. $6^{-2}=$

then $4 a-4 b=$

22. If $\mathrm{a}=-5$, then $\mathrm{a}^{2}=$

23. $\left(\frac{3}{4}\right)^{2}=$

24. $\sqrt{\frac{75}{3}}=$

$25 \cdot 6^{3}=$

$\underline{\text { Solve the linear equations. }}$
26. $3 n+2 n=20$
27. $7(n+13)=42$
28. $\frac{2}{3} w+3=10$
29. $\frac{4+9 y}{12}=\frac{1}{3}$
30. $2 g+9+6+4 g=6\left(g+\frac{5}{2}\right)$
31. $\frac{1}{3} n+\frac{1}{3} n=\frac{2}{5}$
32. $9 x-5=2 x+9$
33. $\frac{1}{3}(x+5)+\frac{2}{3}(x+5)=7$
34. $2(1-3 x)=11$

Solve the systems of equations.

35. $2 x+y=1$

$x+y=3$
36. $3 y+4 x=0$
$y=x-7$

\section{Factor completely}



37. $2 x^{2}-98$
38. $x^{2}+2 x-15$
39. $x^{2}-10 x+25$
40. $6 x^{2}+5 x+1$
41. $2 x^{2}+5 x-3$
42. $4 x^{2}-19 x-5$

Solve the quadratic equations.
43. $5 x^{2}=45$
44. $x^{2}+4=29$
45. $x^{2}+9 x+20=0$
46. $3 x^{2}-5 x-12=0$
47. $x^{2}+3 x=5$
48. $2 x^{2}+x=6$

\section{Graph}

49. $y=3 x-1$

50. $y=-\frac{2}{3} x+5$
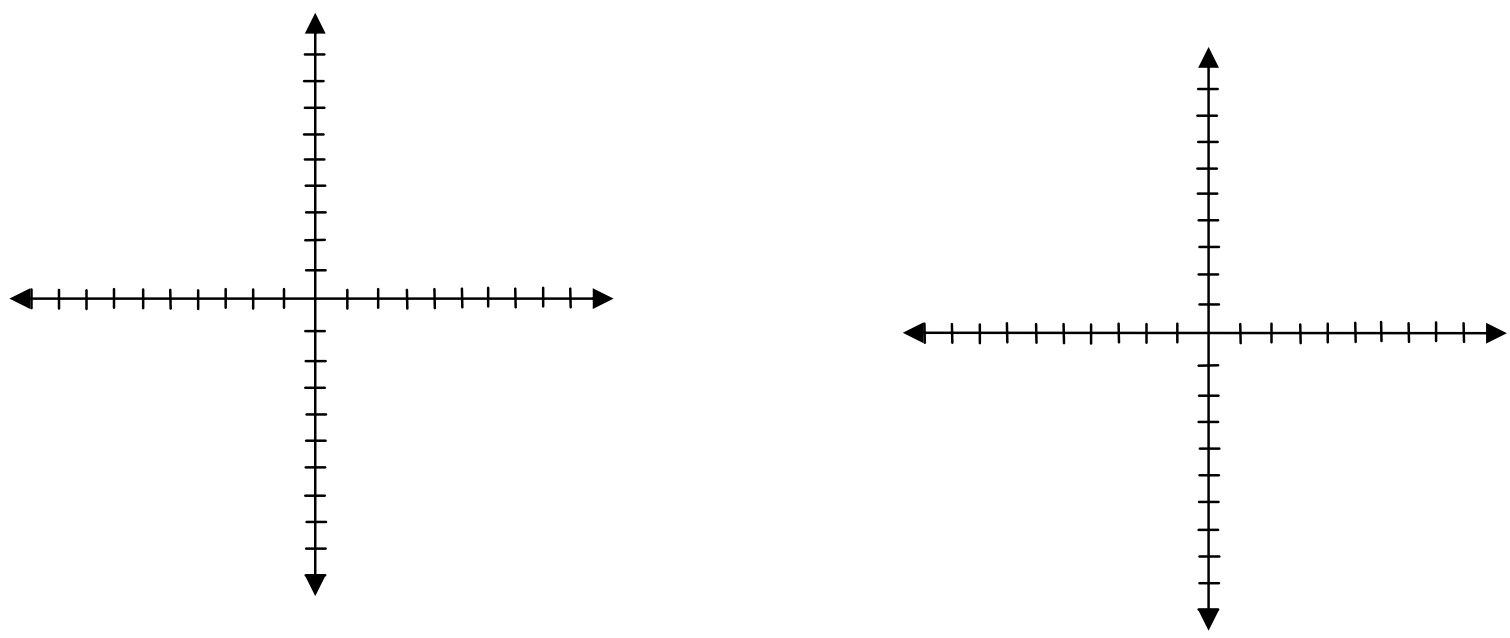

Write the equation 
51. for a line that passes through $(0,-3)$ and $(2,1)$.

52. for a line parallel to $y=2 x+3$ that passes through the point $(5,2)$.

Answer the questions.

53 . What is the vertex of $y=-x^{2}+2 x+5$ ?

54. Which direction will the parabola in \#53 open?

\section{Graph.}

55. $y=x^{2}-4$

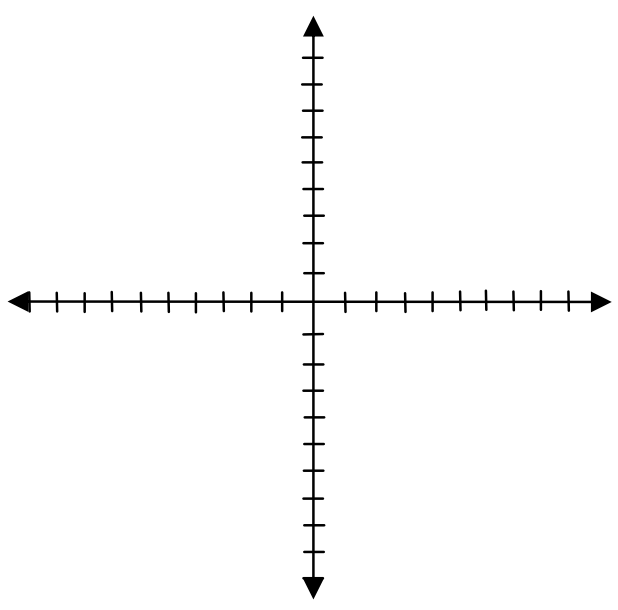

